\title{
RELATOS DE PESQUISAS \\ UMA ARQUITETURA PARA SISTEMAS DE GESTÃO DO CONHECIMENTO ORIENTADA A GRUPOS DE PESQUISA E DESENVOLVIMENTO
}

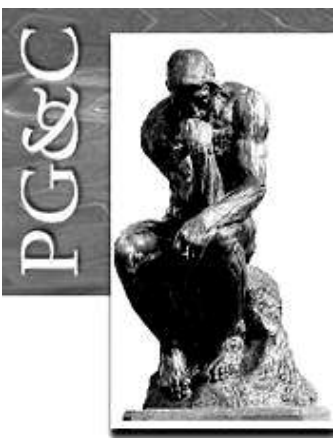

\author{
Olival de Gusmão Freitas Júnior \\ Doutor em Engenharia de Produção pela Universidade Federal de Santa \\ Catarina, Brasil. Professor da Universidade Federal de Alagoas, Brasil. \\ E-mail: olival@ic.ufal.br \\ Victor Diogho Heuer de Carvalho \\ Mestre em Engenharia de Produção pela Universidade Federal de \\ Pernambuco, Brasil. Professor da Universidade Federal de Alagoas, Brasil. \\ E-mail: victor.carvalho@delmiro.ufal.br \\ Petrucio Antonio Medeiros Barros \\ Mestre em Modelagem Computacional do Conhecimento pela \\ Universidade Federal de Alagoas, Brasil. \\ Professor da Universidade Federal de Alagoas, Brasil. \\ E-mail: petrucio.barros@ic.ufal.br \\ Marcus de Melo Braga \\ Doutor em Engenharia e Gestão do Conhecimento pela Universidade \\ Federal de Santa Catarina, Brasil. \\ Professor da Universidade Federal de Alagoas, Brasil. \\ E-mail: marcus@ic.ufal.br
}

\section{Resumo}

Este artigo tem como objetivo apresentar uma arquitetura para Sistemas de Gestão do Conhecimento (SGC), auxiliando na gestão do conhecimento em grupos de pesquisa e desenvolvimento, visando facilitar a criação, a disseminação e o compartilhamento do conhecimento, de forma a beneficiar a qualidade e a produtividade desses grupos. O desenvolvimento dessa arquitetura engloba quatro elementos básicos: a análise e a compreensão do ambiente organizacional complexo das IES, a identificação dos processos de gestão do conhecimento, a criação e a preservação da memória organizacional e a gestão por competências dos membros dos grupos de pesquisa. Para a obtenção da arquitetura, foi utilizada uma metodologia da Engenharia do Conhecimento, que contém uma biblioteca com uma série de componentes essenciais para sua concepção. Por meio da aplicação deste método, obteve-se uma visão esquemática do ambiente e da arquitetura para um SGC destinado a uso pelos grupos de pesquisa, assim como foi possível conceber, através dos elementos levantados, uma arquitetura fundamental para portais do conhecimento a serem utilizados por estes grupos. Os principais esquemas resultantes do processo de modelagem são apresentados neste trabalho.

Palavras-chave: Inteligência Coletiva. Grupos de Pesquisa e Desenvolvimento. Sistemas de Gestão do Conhecimento; Engenharia do Conhecimento. Modelagem de Ambiente. 


\title{
AN ARCHITECTURE FOR KNOWLEDGE MANAGEMENT SYSTEMS ORIENTED TO RESEARCH AND DEVELOPMENT GROUPS
}

\begin{abstract}
This paper aims to present an architecture for knowledge management systems (KMS) that assist research and development groups to facilitate the creation, dissemination, and sharing of knowledge and to improve the quality and productivity of these groups. The development of this architecture consists of four basic elements: the analysis and understanding of the complex organizational environment of HEls, the identification of knowledge management processes, the creation and preservation of organizational memory, and the competency management of the research groups' members. To obtain the architecture, a knowledge engineering method was used, which contains a library with a series of components essentials to its conception. Through this method, a schematic vision of the environment and the architecture for a KMS oriented toward research groups was obtained. Moreover, it was possible to design, through the functionalities identified, a fundamental architecture for knowledge portals that could be used by these groups. The main schemes resulting from the modeling process are presented in this paper.
\end{abstract}

Keywords: Collective Intelligence. Research and Development Groups. Knowledge Management Systems. Knowledge Engineering. Environmental Modeling.

\section{INTRODUÇÃO}

As universidades possuem um papel privilegiado na sociedade do conhecimento, responsabilizando-se pela geração, disseminação e utilização do conhecimento. Desta maneira, torna-se fundamental analisar os aspectos relativos à gestão do conhecimento, visando preservar o conhecimento existente nas Instituições de Educação Superior (IES), bem como gerar novos conhecimentos, fundando-se nos conhecimentos existentes.

Verifica-se um crescimento no fomento à pesquisa nas IES, estimulando a formação de novos pesquisadores e o desenvolvimento de pesquisas nas diversas áreas do conhecimento. Geralmente, a atividade de pesquisa nas IES está diretamente associada aos grupos de pesquisa e desenvolvimento (P\&D) institucionais, que foram construídos considerando as informações presentes na base de dados do Diretório de Grupos de Pesquisa no Brasil (GONÇALVES, 2000; CNPq, 2016). Esses grupos, formados por pesquisadores, objetivam o desenvolvimento de pesquisas que contribuam para o avanço no campo científico ou tecnológico.

Os grupos de pesquisa e desenvolvimento (P\&D) tornam-se cada vez mais importantes nas IES, tanto em termos de direcionamento das pesquisas institucionais quanto para reconhecimento por órgãos externos. Esses grupos geram e acumulam, ao longo do tempo, grande quantidade de ativos de informação e conhecimento, por meio de projetos de pesquisa, publicação de trabalhos científicos e tecnológicos, redes de pessoas e demais fontes.

Igualmente, verifica-se que algumas características presentes em grupos de pesquisa (elevada rotatividade de pesquisadores, limitação de recursos financeiros, evolução contínua do conhecimento, informalidade na comunicação, membros geograficamente dispersos, entre outros fatores) dentro de uma IES evidenciam ainda mais a necessidade de gerenciar o conhecimento, visando favorecer a produtividade e a maximização de resultados.

Diante disso, constata-se que, para obter sucesso na atividade de pesquisa em uma IES, é fundamental desenvolver a capacidade de gerenciar sistematicamente o conhecimento gerado nos grupos de pesquisa. Nesses grupos, o conhecimento deve ser intensamente compartilhado e gerenciado, por ser a principal matéria-prima na geração de ideias e

Perspectivas em Gestão \& Conhecimento, João Pessoa, v. 7, Número Especial, p. 126-144, mar. 2017. 
resultados. Essa premissa torna-se cada vez mais verdadeira na medida em que os referidos grupos tornam-se multidisciplinares e com seus pesquisadores atuando em rede. Além disso, é necessário enfatizar a importância do aprendizado colaborativo e cooperativo em rede, como forma de incrementar o potencial de inteligência coletiva dos pesquisadores pertencentes aos grupos de pesquisa, bem como enfatizar a necessidade de que o conhecimento necessário à realização das atividades desenvolvidas nesses grupos seja gerenciado de forma sistemática.

O objetivo deste artigo é apresentar uma arquitetura para Sistemas de Gestão do Conhecimento (SGC) para auxiliar a gestão do conhecimento em grupos de pesquisa, visando facilitar a criação, a disseminação e o compartilhamento do conhecimento, de forma a beneficiar a qualidade e a produtividade desses grupos.

Sua sequência está dividida da seguinte forma: a seção 2 trabalha o referencial teórico com base em três temas centrais - fundamentos sobre grupos de P\&D, gestão do conhecimento em grupos de P\&D e inteligência coletiva; a seção 3 apresenta a metodologia de pesquisa; a seção 4 apresenta e discute um modelo de arquitetura para SGCs orientada a grupos de P\&D; e, por fim, a seção 5 apresenta as considerações finais desta pesquisa.

\section{REFERENCIAL TEÓRICO}

\subsection{Fundamentos sobre Grupos de Pesquisa e Desenvolvimento}

Normalmente, a atividade de pesquisa nas IES está vinculada aos grupos de P\&D institucionais. Esses grupos estão organizados em torno da execução de linhas de pesquisa, e seus pesquisadores estão envolvidos com a realização de projetos de pesquisa e com a produção científica e tecnológica.

Silva et al. (2003) utilizam a conceituação de Karl Erik Sveiby ao definir que um grupo de pesquisa é formado por um conjunto de indivíduos que têm interesses semelhantes sob um determinado tema, ou seja, pessoas que mantêm interesse comum numa tecnologia, produtos ou áreas, visando ao aprendizado e ao desenvolvimento pessoal.

De acordo com Gonçalves (2000, p.32), um "grupo de pesquisa pode ser visto como a união de uma ou mais pessoas, organizadas hierarquicamente, com objetivos em determinadas linhas de pesquisa e sob a liderança de um ou dois líderes".

Segundo Romão (2002), um grupo de pesquisa é caracterizado por um ou dois pesquisadores que atuam em uma ou mais linhas de pesquisa, compartilhando recursos comuns. Além disso, não é obrigatório que os integrantes de um grupo pertençam a uma única instituição. Para Agrasso Neto e Abreu (2002a, p. 4), um "grupo de pesquisa consiste em um ou mais indivíduos que são psicologicamente conscientes dos papéis exercidos e que interagem para atingir uma meta comum".

Considerando os diversos conceitos apresentados, é útil e necessário conceituar o termo "grupo de pesquisa" que será adotado neste trabalho. Trata-se de um grupo de pesquisadores que se organiza em torno da execução de linhas de pesquisa, segundo uma regra hierárquica fundamentada na experiência e na competência técnico-científica, compartilhando recursos comuns e possuindo dois objetivos principais: (1) o desenvolvimento de pesquisas que contribuam para o avanço no campo científico ou tecnológico e (2) formação de novos pesquisadores.

Os grupos de pesquisa estão localizados em universidades, instituições isoladas de Ensino Superior, institutos de pesquisa científica, institutos tecnológicos, laboratórios de pesquisa e desenvolvimento de empresas estatais ou ex-estatais e em algumas organizações não governamentais com atuação em pesquisa (ROMÃO, 2002). Esses grupos foram construídos considerando as informações presentes na base de dados do Diretório de Grupos

Perspectivas em Gestão \& Conhecimento, João Pessoa, v. 7, Número Especial, p. 126-144, mar. 2017. 
de Pesquisa no Brasil, cujo objetivo é oferecer um suporte atualizado das atividades de pesquisa.

Segundo o CNPq (2016), grupo de pesquisa pode ser definido como um conjunto de indivíduos organizados hierarquicamente de acordo com sua competência no campo científico ou tecnológico, existindo envolvimento profissional e permanente em atividades de pesquisa. Nesses grupos, o trabalho organiza-se em torno de linhas comuns de pesquisa e seus componentes, em algum grau, compartilham recursos.

Geralmente, um grupo de pesquisa necessita satisfazer duas funções básicas: funções de tarefa e funções de manutenção. As funções de tarefa correspondem aos atos e comportamentos relacionados à produtividade e dirigidos à realização das atividades do grupo. Essas funções refletem o aspecto racional e operacional das atividades a serem realizadas pelo grupo para o atendimento de metas e objetivos. As funções de manutenção referem-se aos atos e comportamentos que exprimem as necessidades emocionais e sociais dos membros do grupo. A ênfase está em satisfazer essas necessidades, bem como em estabelecer e manter relações interpessoais de cooperação, coesão e uma atitude favorável em relação ao grupo (BOWDITCH; BUONO, 1992).

Ambas as funções são essenciais para a eficácia do grupo de pesquisa. Assim, um grupo eficaz é aquele que apresenta desempenho na tarefa e manutenção dos recursos humanos. O desempenho na tarefa refere-se ao alcance de metas de desempenho, levando em consideração os custos, os prazos, os recursos e a qualidade técnica. A manutenção dos recursos humanos considera se os membros do grupo estão suficientemente satisfeitos com suas tarefas, os resultados e o relacionamento interpessoal para que trabalhem de maneira coesa.

Observa-se que os grupos de pesquisa são constituídos por projetos de pesquisa que deverão estar ligados por uma linha de pesquisa, permitindo a cada projeto desenvolver o seu objeto de pesquisa, a fim de contribuir para o fortalecimento de sua linha. Assim, linhas de pesquisa representam temas aglutinadores de estudos científicos que se fundamentam em tradição investigativa, na qual se originam projetos de pesquisa cujos resultados guardam afinidades entre si (CNPq, 2016).

A norma ISO 10006 de 2003 define projeto como um grupo de atividades coordenadas e controladas, com data para início e término, empreendido para o alcance de um objetivo comum, conforme requisitos específicos, incluindo limitações de tempo, custo e recursos (ISO, 2003). Esses elementos constituem a estrutura de um grupo de pesquisa (Figura 1).

Figura 1 - Estruturação de um grupo de pesquisa

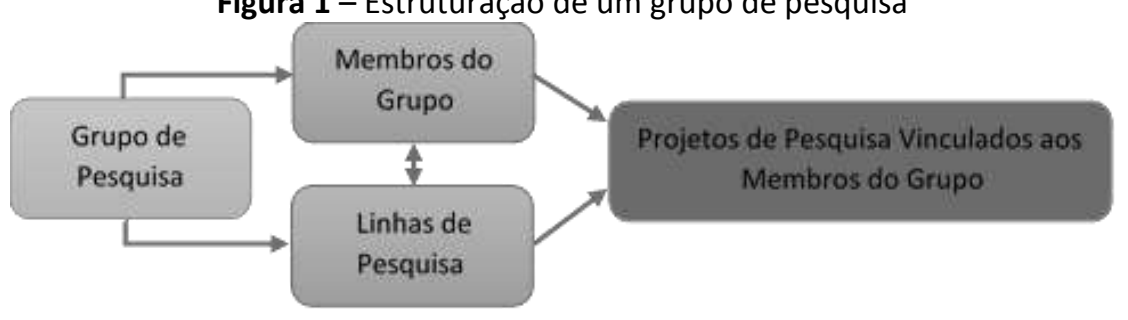

Os pesquisadores dos grupos de pesquisa necessitam possuir conhecimentos relacionados a áreas específicas, de maneira que possam desenvolver eficaz e eficientemente seu trabalho. Esse conhecimento é formado considerando os integrantes do grupo que contribuem com suas experiências e habilidades e com seus conhecimentos especializados (AGRASSO NETO; ABREU, 2002b).

Segundo Agrasso Neto e Abreu (2002b), grupos de pesquisa são caracterizados por utilizar tecnologias avançadas relacionadas às áreas de atuação dos seus pesquisadores. Esse fato é evidenciado principalmente quando as áreas de atuação de um grupo de pesquisa 
envolvem aspectos multidisciplinares, reunindo pesquisadores que se propõem a dialogar e trocar seus conhecimentos, visando à solução de problemas.

Desta forma, os grupos de pesquisa possuem características próprias. Sua dinâmica é determinada por normas e expectativas de seus pesquisadores e pelos mecanismos de apoio de que dispõem - que, se bem definidos e gerenciados, possibilitarão direcionar esforços na busca do alto desempenho de seus membros. Segundo Lichtnow (2001), Bowditch e Buono (1992), podem ser observadas as seguintes características nos grupos de pesquisa:

- Diferentes níveis de conhecimento. No interior de um grupo de pesquisa, existem membros que estão vinculados há mais tempo. Esses, normalmente, possuem diferenças de tempo de vinculação ao grupo e de conhecimentos com relação às linhas de pesquisa (pelo menos no que se refere à área que é objeto de estudo dentro do grupo). Tais diferenças podem também ser fruto da formação com ênfase diferenciada dos membros do grupo;

- Tratamento inadequado do conhecimento gerado. Apesar de o conhecimento estar presente dentro de um grupo de pesquisa, muitas vezes não recebe o tratamento adequado, ou seja: esse conhecimento ou não está registrado, ou está registrado de forma inadequada ou não se encontra facilmente acessível;

- Evolução contínua do conhecimento. Um grupo de pesquisa precisa lidar com a evolução contínua do conhecimento e com os rápidos avanços tecnológicos. Isso requer que o conhecimento armazenado seja constantemente avaliado ou atualizado, reunindo novas experiências e conhecimentos a cada vez que uma nova atividade é realizada;

- Informalidade na comunicação. O relacionamento entre os membros de um grupo de pesquisa tende a ser bastante informal. Em outras palavras, o conhecimento coletivo, especialmente aquele que propicia a integração de novos membros, é transferido por meio de conversas e observação do uso que os mais experientes fazem. $O$ resultado disso é a quase inexistência de documentação de algumas das soluções adotadas;

- Repetições de erros. Uma vez que as tarefas realizadas frequentemente não são documentadas, as lições aprendidas não são consideradas e registradas. Desta forma, na busca por soluções, tentativas fracassadas são novamente testadas. Também é observado com frequência que, em função de perda de informações, soluções que já foram desenvolvidas são desenvolvidas novamente;

- Limitação de recursos financeiros e humanos. Os grupos de pesquisa convivem com poucos recursos (financeiros e humanos) para sua manutenção, sendo, então, necessária uma alocação eficiente dos recursos existentes;

- Definição de normas de comportamento. Normas são padrões ou ideias comuns que norteiam o comportamento dos membros de um grupo. Embora, em alguns casos, as normas possam ser formalizadas e escritas, na maioria das vezes há regras não escritas relativas a esses comportamentos, que são apropriadas e aceitas pelos demais membros do grupo;

- Papéis claramente definidos. O conceito de papel refere-se aos diversos comportamentos que os membros devem desempenhar dentro do grupo. Essas expectativas comportamentais são influenciadas pela localização de um papel na hierarquia do grupo, pelas atividades associadas a esse papel e pelos seus padrões de interação social, o que determina a série de comportamentos tidos como aceitáveis;

- Liderança. É necessário dispor de uma liderança capaz de influenciar o alinhamento entre os objetivos pessoais e os objetivos do grupo e de capacitar os membros a trabalharem de modo cooperativo e autogerenciado para alcançar tais objetivos. 0 
relacionamento interpessoal entre o líder e os membros do grupo é um dos fatores mais relevantes na criação de um clima de confiança, respeito e afeto, possibilitando relações de harmonia e cooperação;

- Elevado turnover. Normalmente, um grupo de pesquisa é formado por membros permanentes e temporários. Os membros permanentes são representados pelos docentes e técnicos. Os membros temporários, formados pelos estudantes, permanecem durante um determinado período de tempo no grupo, tempo esse que corresponde geralmente à duração dos seus cursos de graduação e pós-graduação. Desta forma, as experiências dos membros temporários devem ser compartilhadas com os outros integrantes do grupo, visando minimizar o efeito do turnover e ampliar o conhecimento organizacional;

- Membros geograficamente dispersos. É comum um grupo de pesquisa agregar o conhecimento e a capacidade de membros que, muitas vezes, encontram-se geograficamente dispersos, o que enfatiza ainda mais a necessidade da existência de uma infraestrutura tecnológica capaz de favorecer a colaboração e a cooperação de seus membros;

- Cooperação entre várias comunidades. Geralmente, o trabalho em grupos de pesquisa envolve a interação de comunidades distintas. Diferentes pesquisadores juntam-se ao grupo, possuindo diferentes pontos de vista, interesses e conhecimentos, viabilizando as discussões interdisciplinares e multidisciplinares.

Essas características, bastante presentes nos grupos de pesquisa em uma IES, geram necessidades que deverão ser supridas - se não em sua totalidade, pelo menos parcialmente por um ambiente que forneça suporte à gestão do conhecimento. Além disso, tais particularidades dificilmente podem ser alteradas de forma significativa, restando a esses grupos a alternativa de criar iniciativas para reter e gerenciar seus conhecimentos, o que refletirá diretamente no processo de ensino-aprendizagem.

As atividades desenvolvidas em um grupo de pesquisa envolvem um conhecimento altamente especializado, além de facilidade de acesso às novas informações. De acordo com Lichtnow (2001), as principais atividades realizadas dentro de um grupo de pesquisa que podem ser suportadas por meio de conhecimento, experiência e habilidade são:

- Cooperação de pesquisa e/ou elaboração de projetos de pesquisa. Essa atividade requer conhecimento sobre programas de pesquisa e, também, conhecimentos que auxiliem no planejamento e na execução de projetos de pesquisa;

- Desenvolvimento de modelos conceituais e construção de protótipos. Essa atividade requer o desenvolvimento de modelos conceituais para posterior desenvolvimento de um protótipo. Os pesquisadores estruturam, categorizam, ordenam e generalizam suas experiências e observações em termos de conceitos. Para descrevê-los, utilizamse definições conceituais, que são aplicadas frequentemente para organizar os conceitos. Assim, alguns projetos de pesquisa demandam a construção de protótipos, visando ajustar as funcionalidades das soluções implementadas, antecipando problemas potenciais;

- Elaboração e publicação de trabalhos científicos. Essa atividade consiste na elaboração de trabalhos científicos que instrumentalizam as ações de ensino e pesquisa ou que são resultantes delas. Assim, essa atividade requer conhecimento quanto ao estado da arte e procedimentos metodológicos a serem utilizados na elaboração desses trabalhos. Inclui relatórios técnicos, artigos, dissertações e teses; 
- Participação em cursos e eventos. Essa atividade consiste em manter informações atualizadas sobre cursos e eventos de interesse do grupo (conferências, simpósios, congressos, workshops etc.) a serem realizados;

- Pesquisa e revisão da literatura. Essa atividade requer pesquisa e revisão da literatura relevante ao suporte das atividades de pesquisa realizadas pelos membros do grupo;

- Realização de reuniões para apresentação e discussão de ideias. Essa atividade necessita de suporte organizacional quanto ao gerenciamento das reuniões, além de conhecimento sobre preparação e condução dessas atividades. As reuniões são realizadas com uma variedade de propósitos, tais como: reuniões de estudo, reuniões para tomada de decisões, discussão de problemas, solução de conflitos, elaboração de projetos, entre outros aspectos;

- Aprendizagem. Essa atividade enfatiza a necessidade da utilização de tutoriais e de material relacionado às áreas de atuação do grupo que possam favorecê-la;

- Desenvolvimento de novos projetos de pesquisa. Essa atividade requer conhecimento em relação às características dos projetos de pesquisa e aos requisitos envolvidos em seu desenvolvimento e em sua execução, além da existência de templates e de um banco de projetos aprovados.

Observa-se, assim, que as atividades desenvolvidas nos grupos de pesquisa requerem conhecimentos especializados e facilidades de acesso a novas informações, conhecimentos e tecnologias. Essas premissas apresentam-se cada vez mais verdadeiras, à medida que os referidos grupos se tornam multidisciplinares e com seus pesquisadores atuando em rede.

A análise dessas atividades enfatiza também a necessidade de que o conhecimento necessário à sua realização seja gerenciado de forma sistemática dentro dos grupos de pesquisa, além de demonstrar claramente os benefícios que tal gerência pode trazer para a realização das atividades.

\subsection{A Gestão do Conhecimento em Grupos de Pesquisa e Desenvolvimento}

Verifica-se que os conceitos, técnicas e práticas de gestão do conhecimento podem auxiliar no desafio de geração de resultados dentro dos grupos de pesquisa, envolvendo múltiplos agentes e ações e que devem ser tratados de forma estratégica nas IES. Assim, a gestão do conhecimento em grupos de pesquisa exige a valorização de iniciativas que agreguem valor e tragam resultados, visando avaliar a contribuição efetiva das ações que estão sendo desenvolvidas dentro desses grupos (SILVA, 2003).

Outrossim, reconhece-se que as atividades desenvolvidas nos grupos de pesquisa requerem conhecimentos especializados e facilidades de acesso a novas informações e que os grupos de pesquisa possuem certas características que, se bem definidas e gerenciadas, poderão direcionar esforços na busca do alto desempenho de seus integrantes. Entre as características apresentadas, algumas evidenciam a necessidade da gestão do conhecimento em grupos de pesquisa: (i) tratamento inadequado do conhecimento gerado; (ii) existência de diferentes níveis de conhecimento; (iii) limitação de recursos financeiros e humanos; (iv) informalidade das comunicações; (v) contínua evolução do conhecimento e (vi) elevada rotatividade dos membros (turnover).

Desta forma, percebe-se a necessidade de se reter e compartilhar o conhecimento dos membros que compõem o grupo, de modo a torná-lo parte da memória organizacional. Tratase, portanto, de estruturar e armazenar o conhecimento individual, disponibilizando-o por todo o grupo de pesquisa (LICHTNOW, 2001; SILVA et al., 2003).

Perspectivas em Gestão \& Conhecimento, João Pessoa, v. 7, Número Especial, p. 126-144, mar. 2017. 
Assim, a gestão do conhecimento em um grupo de pesquisa pode gerenciar sistematicamente o conhecimento existente. Esse conhecimento ocorre na forma explícita, estando ligado aos procedimentos, aos bancos de dados e, especialmente, aos documentos produzidos pelo grupo; existe, no entanto, também na forma tácita, representado pelas experiências e habilidades de seus membros.

Garcia e Valentim (2014) destacam algumas especificidades da dinâmica do conhecimento científico para o ambiente universitário, entre eles: a literatura especializada e os eventos da área são as fontes de informação mais usadas; a cultura organizacional é um fator fundamental para a geração, apropriação e socialização do conhecimento científico; diretrizes e políticas institucionais são importantes, principalmente para a geração e a socialização do conhecimento científico, entre outras particularidades.

As condições para que ocorra o processo de gestão do conhecimento em um grupo de pesquisa são: (i) estruturar o grupo para que aconteça a troca de conhecimento entre os seus membros; (ii) definir processos que envolvam a criação, a disseminação e o compartilhamento do conhecimento; (iii) identificar, representar e modelar o conhecimento relevante para o grupo e (iv) identificar que ferramentas podem auxiliar no processo de gerência do conhecimento.

Para atender a essas necessidades identificadas nos grupos de pesquisa institucionais, é necessária a adoção de uma infraestrutura de suporte à gestão do conhecimento que proporcione os seguintes benefícios:

- A contínua criação do conhecimento, por meio de atividades de pesquisa realizadas pelos membros do grupo;

- O constante compartilhamento desse conhecimento entre os seus integrantes;

- O constante acompanhamento do nível de conhecimento de cada indivíduo do grupo;

- A facilidade de acesso, geração, estruturação, disseminação e utilização das informações, provendo o contexto de conhecimento necessário para a tarefa a ser executada.

É evidente que a gestão do conhecimento em grupos de pesquisa deve estar baseada em um modelo de sistema de gestão do conhecimento compatível com as características desses grupos.

\subsection{A Inteligência Coletiva e sua Influência nos Grupos de Pesquisa}

A palavra inteligência origina-se do latim inter (entre) e legere (escolher), ou seja, significa aquilo que permite ao ser humano escolher entre uma coisa e outra. Inteligência é a habilidade de realizar de maneira eficiente uma determinada tarefa (FERNANDES, 2003).

Segundo Lévy (1997), a inteligência coletiva surge da necessidade de empregar pessoas capazes de tomar iniciativas, resolver problemas, propor novas soluções, de forma coletiva e organizada, visando à construção de um saber comum. Além disso, a inteligência coletiva reside nos processos pelos quais as pessoas se influenciam mutuamente e que se verificam considerando o equilíbrio entre competição e cooperação.

$\mathrm{Na}$ inteligência coletiva, há a valorização, a utilização otimizada e a colocação em sinergia das competências e do raciocínio de forma participativa e colaborativa por muitos indivíduos. Esse ideal da inteligência coletiva passa, evidentemente, pela colocação em comum da memória de experiência por uma prática de intercâmbio/compartilhamento de conhecimentos (CINALLI et al., 2015).

Perspectivas em Gestão \& Conhecimento, João Pessoa, v. 7, Número Especial, p. 126-144, mar. 2017. 
De acordo com Lévy (2002), pode-se analisar a inteligência coletiva por meio de quatro dimensões básicas: a dimensão técnica, a dimensão cultural, a dimensão social e a dimensão intelectual. As inter-relações entre essas dimensões podem ser visualizadas na Figura 2.

Figura 2 - As quatro dimensões da inteligência coletiva

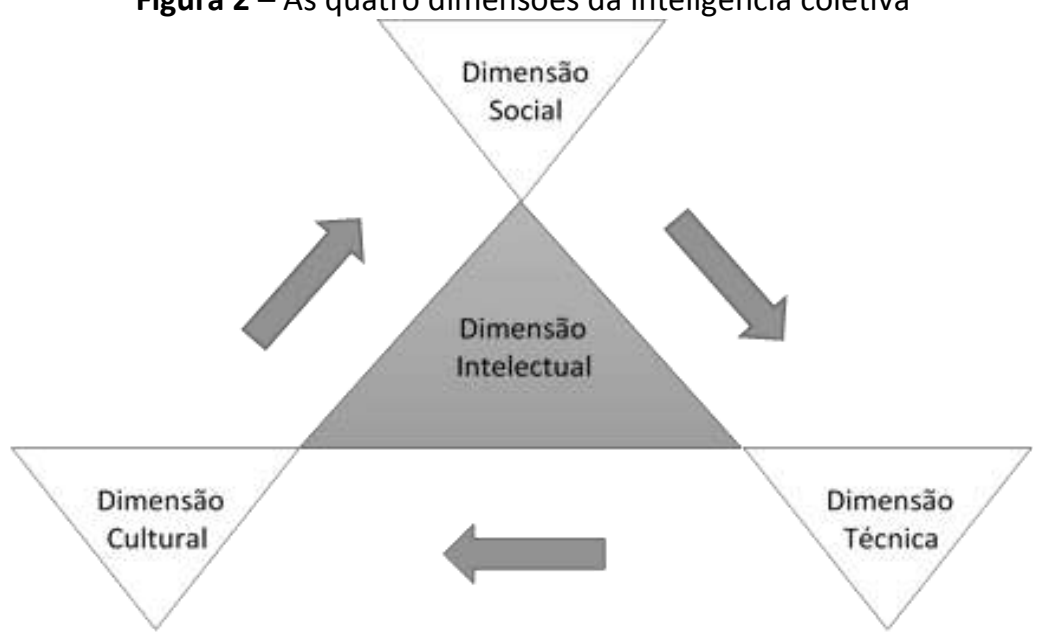

Fonte: Lévy (2002)

A primeira dimensão - técnica - fornece a tecnologia da informação e a comunicação necessárias para as pessoas compartilharem e disseminarem experiências e ideias. $O$ desafio dessa dimensão consiste em identificar tecnologias que apoiem a comunicação e a troca de conhecimentos e habilidades, facilitando a colaboração entre os indivíduos e incentivando as pessoas a se unirem, a participarem, a tomarem parte em grupos e comunidades e a renovarem suas competências. As pessoas, fazendo uso da internet, podem compartilhar conhecimentos e experiências, formando redes de cooperação (LÉVY, 2002).

Os grupos de pesquisa utilizam diferentes dimensões de trabalho, adotando diversas tecnologias. Cada tecnologia adotada cria um tipo de interdependência que, por sua vez, exige certo de tipo de coordenação e tem considerações estruturais diferentes. Entre as tecnologias pertencentes a essa dimensão, encontram-se: internet, intranet, groupware, suportes de memória e de percepção coletiva. O papel dessa dimensão é ajudar o desenvolvimento do conhecimento coletivo e do aprendizado contínuo, tornando mais fácil para os membros compartilharem problemas, perspectivas, ideias e soluções no interior de um grupo de pesquisa, possibilitando, ainda, que haja um incremento do potencial inovador e de transferência tecnológica do grupo, uma educação/formação continuada de seus participantes e engajamento do ponto de vista da dimensão social, discutida um pouco adiante (SECUNDO et al., 2016).

A segunda dimensão - cultural - refere-se ao conteúdo e à organização da estrutura de ideias, que são visualizadas e representadas por signos. Essa dimensão representa o conjunto dos conhecimentos produzidos que estão armazenados na memória (LÉVY, 2002). O objetivo da memória é favorecer o crescimento, a transmissão e a conservação dos conhecimentos dentro de uma organização de forma dinâmica. Além disso, a dimensão técnica oferece as condições para o aprimoramento da dimensão cultural.

Os grupos de pesquisa trabalham com diversos tipos de informação ou conhecimento, relacionados a diversas áreas do conhecimento, contemplando vários objetivos (realização de pesquisas, resolução de problemas, formação de pesquisadores e outros) e levando em consideração pontos de vista diferentes. O papel da dimensão cultural consiste em enfatizar o aprendizado cooperativo e colaborativo em rede, como forma de incrementar o potencial de 
inteligência coletiva dos grupos de pesquisa. Além disso, a memória organizacional, suportada por processos de gestão do conhecimento e por tecnologias orientadas a esta finalidade (memória sociocultural), fornece ativamente ao membro de um grupo o conhecimento (ou informação) requerido para cumprir a tarefa (LÉVY, 2010).

A terceira dimensão - social - refere-se às relações, aos vínculos de intercâmbios de serviços, de conhecimento e de sociabilidade. Essa dimensão enfatiza os vínculos entre pessoas e, particularmente, as qualidades desses vínculos. Tal ênfase influencia na cooperação intelectual, que é importante para o desenvolvimento cultural e social (LÉVY, 2002).

Em um grupo de pesquisa, há diversos tipos de relacionamento entre os seus membros. Os grupos de pesquisa estão voltados para a realização de tarefas, nas quais seus integrantes interagem diretamente para o cumprimento de suas metas e objetivos. Todavia, os grupos de pesquisa devem levar em consideração as necessidades sociais e emocionais de seus membros em favor da realização da tarefa. Assim, nos grupos de pesquisa, a ênfase está em estabelecer e manter relações interpessoais de cooperação, coesão e uma atitude favorável em relação ao grupo. Quando os membros de um grupo identificam seus objetivos particulares com os do grupo, liberam uma enorme quantidade de energia, criatividade e lealdade, despertando também, conforme Tjornbo (2016), um enorme potencial para inovação em suas práticas internas, uma vez que a socialização do conhecimento é incentivada.

A última dimensão - intelectual - corresponde ao somatório das competências e ideias de todos na organização (LÉVY, 2002). As competências e ideias originais formam a dimensão intelectual alimentada pelas outras dimensões - que, por sua vez, são alimentadas por ela. Assim, verifica-se que a dimensão intelectual corresponde à soma do conhecimento de todos na organização. Nesse momento, as organizações podem criar, organizar, disseminar, compartilhar e utilizar o conhecimento gerado, permitindo desenvolver novas competências, criar novos produtos e serviços e demais possibilidades.

Cabe ao grupo de pesquisa estimular o desenvolvimento dessa dimensão como forma de melhorar continuamente a capacitação e a interação coletiva dos integrantes do grupo, fato que é ressaltado por Meza et al. (2015) no âmbito da inteligência colaborativa aplicada à educação. Assim, os grupos de pesquisa necessitam gerenciar e mapear as competências de seus membros, bem como identificar as áreas de conhecimento relacionadas às atividades do grupo que favoreçam a produtividade e a maximização de resultados.

\section{METODOLOGIA}

O desenvolvimento da pesquisa para obtenção de uma arquitetura para SGCS orientada a grupos de P\&D seguiu as premissas da Design Science Research Methodology (DSRM), que provê um fluxo padrão de atividades para processos de pesquisa, fornecendo aos pesquisadores um modelo a ser seguido para a obtenção dos resultados envolvidos em suas investigações (HEVNER et al., 2004). Através da DSRM, são definidas as seguintes atividades para a obtenção do desenho do processo de pesquisa: 1 . Identificação do problema e motivação; 2. Definição dos objetivos para solução; 3. Desenho/projeto e desenvolvimento; 4. Demonstração; 5. Avaliação; e 6. Comunicação (PEFFERS et al., 2007). A aplicação do DSRM neste trabalho, portanto, pode ser parametrizada da seguinte forma:

1. Identificação do problema e motivação: aplicação de conceitos relacionados à gestão da informação e do conhecimento e carência de ambientes sistêmicos e virtuais para apoiar as atividades de grupos de P\&D. Para apoiar a identificação do problema desta pesquisa, realizou-se uma pesquisa bibliográfica que apontou diversos elementos relacionados a grupos de P\&D e à aplicação da gestão do conhecimento a estes grupos, demonstrando os conceitos relacionados à inteligência coletiva de Pierre Lévy; 
2. Definição dos objetivos: como destacado anteriormente, o objetivo definido foi a criação de uma arquitetura para SGCs orientada a grupos de P\&D;

3. Desenho/projeto e desenvolvimento: para o desenvolvimento do modelo de sistema de gestão do conhecimento em grupos de pesquisa, utilizou-se como referência o método de resolução de problemas (PSM - Problem-Solving Method) CommonKADS, uma vez que ele pode ser empregado como guia para aquisição, representação, modelagem e manutenção do conhecimento, possibilitando o entendimento completo do processo de gestão do conhecimento (SCHREIBER et al., 2000).

4. Demonstração: ao longo da seção 4 deste trabalho, é feita a apresentação dos resultados obtidos por meio da modelagem;

5. Avaliação: ainda ao longo da seção 4, acompanhando a apresentação dos resultados da modelagem, é feita uma discussão em vias de realizar uma avaliação preliminar do modelo obtido;

6. Comunicação: escolha da mídia para publicação do modelo obtido.

Sintetizamos as atividades da pesquisa aplicada para gerar este trabalho em quatro momentos, conforme a Figura 3 a seguir:

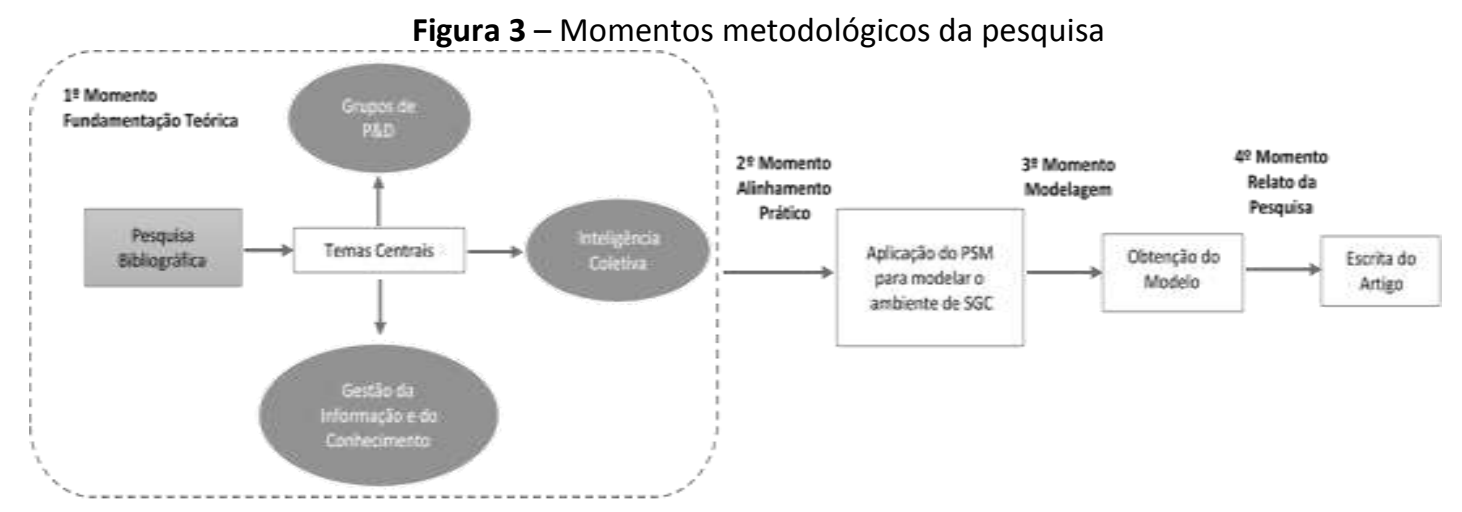

No primeiro momento, foi trabalhada a fundamentação teórica em cima dos três temas centrais, correspondendo à primeira atividade descrita no DSRM. No segundo momento, foi feito o alinhamento prático entre os conceitos teóricos e as necessidades para o desenvolvimento do modelo de arquitetura para SGCs orientada a grupos de P\&D, evidenciando os objetivos a serem alcançados em alinhamento com a segunda atividade do DSRM.

Este segundo momento tornou possível a aplicação posterior do PSM para a criação do modelo, sendo o terceiro momento da pesquisa a própria concepção e obtenção do modelo (processo de modelagem), alinhadas à terceira atividade do DSRM. Com a obtenção do modelo, pôde-se realizar sua descrição. Encontramos o alinhamento com a quarta atividade.

O quarto momento diz respeito ao relato da pesquisa, em forma de artigo, onde é feita uma avaliação preliminar em forma de discussão junto à apresentação do modelo (quinta atividade do DSRM). Por fim, é obtido o artigo completo contendo todo o relato de pesquisa, desde o referencial teórico até a apresentação e a discussão sobre o modelo (sexta atividade).

\section{UMA ARQUITETURA PARA SISTEMAS DE GESTÃO DO CONHECIMENTO ORIENTADA A GRUPOS DE PESQUISA E DESENVOLVIMENTO}

O processo de modelagem da arquitetura para SGCs orientada a grupos de P\&D teve como base a aplicação de um PSM - o CommonKADS. Os PSM podem ser definidos como 
métodos da engenharia do conhecimento que descrevem os componentes de raciocínio para sistemas baseados em conhecimento como padrões de comportamento que podem ser reutilizados entre aplicações de software (FENSEL; MOTTA, 2001).

Dentre os PSM mais conhecidos, podemos destacar: DESIRE, MIKE, MOKA, Protégé II, VITAL e o CommonKADS. Cada um deles possui uma estrutura de aplicação diferente e que não será alvo de discussão neste trabalho. Para tanto, os trabalhos de Studer et al. (1998) e Brazier e Wijngaards (2005) podem ser consultados. Optou-se pelo CommonKads devido à intimidade dos autores com essa metodologia e por ser um padrão reconhecido para o desenvolvimento de sistemas como o aqui proposto (BREUKER; VAN DE VELDE, 1994).

O modelo de SGC para grupos de pesquisa possui como infraestrutura central a memória organizacional e um conjunto de atividades de gestão do conhecimento, a saber: criação, identificação e aquisição, validação, codificação, disseminação e manutenção do conhecimento. Essas atividades podem ser realizadas considerando a identificação do objeto de conhecimento, e cada uma delas possui ferramentas específicas para descoberta, filtragem, busca, distribuição e associação do conhecimento.

Para o seu desenvolvimento, foi proposto um ambiente composto por diferentes ferramentas e agentes, no qual cada agente possuirá um perfil de interação com esses instrumentos (Figura 4).

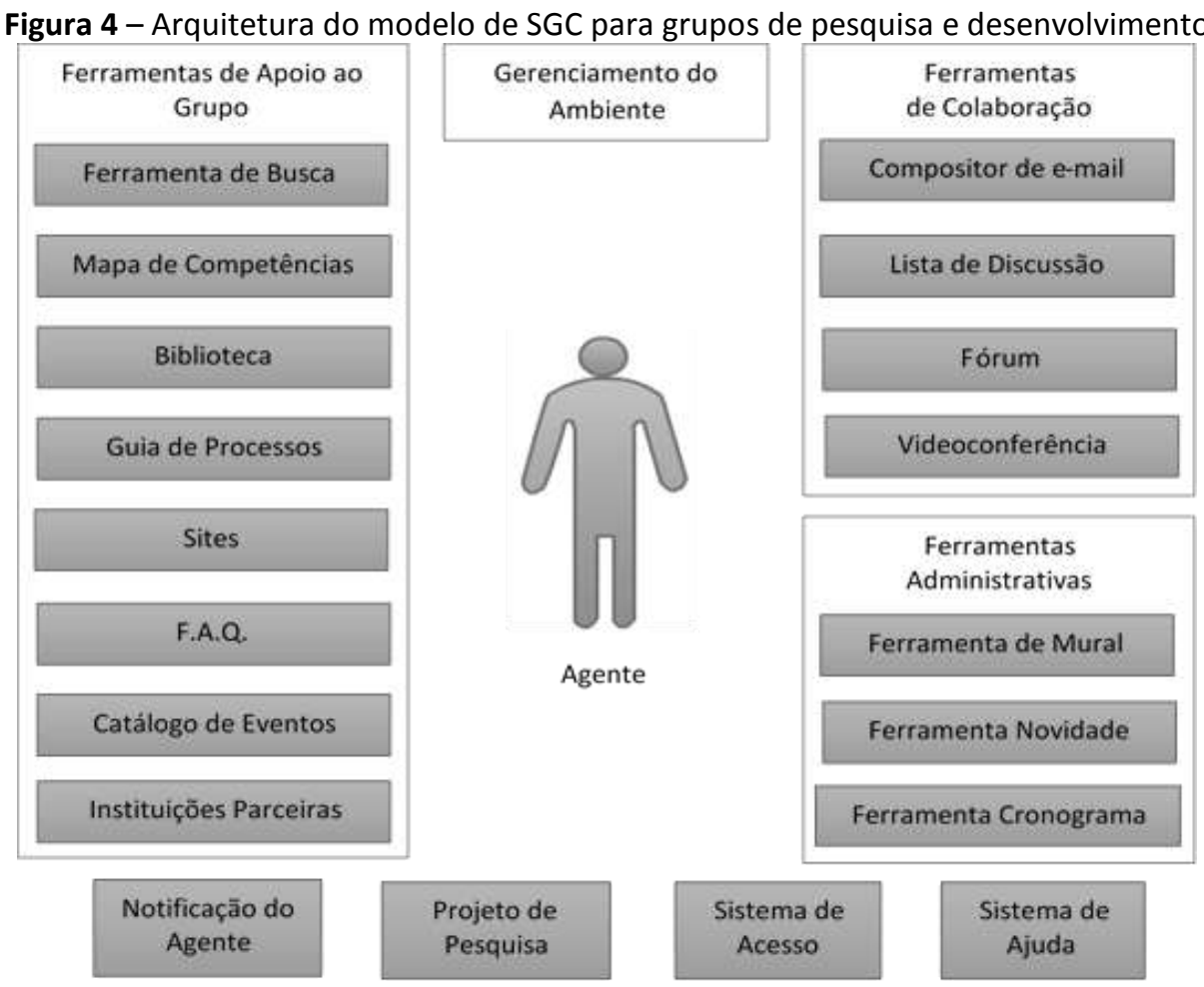

Fonte: Autoria própria

A seguir, será apresentada uma visão individualizada dos agentes e das funcionalidades disponíveis, assim como uma visão de cada funcionalidade.

1. Usuários do ambiente - usuários são agentes que interagem com o ambiente. Seu acesso ao ambiente é definido por meio de perfis e permissões. Cada categoria de usuário tem um ambiente personalizado adequado às suas necessidades e permissões. Os perfis e as permissões são especificados no instante do cadastramento do usuário e personalizados no momento do seu login no ambiente. Os usuários têm acesso às 
ferramentas do ambiente e possuem um assistente pessoal personalizado, de acordo com sua categoria: (i) Líder - usuário que tem permissão para tomar decisões que afetam o trabalho do grupo de pesquisa. Esse usuário pode contribuir na coleta, avaliação e manutenção da memória organizacional. Acessa as ferramentas de apoio ao grupo, ferramentas administrativas, ferramentas de colaboração, gerenciamento do ambiente, sistema de acesso, sistema de ajuda e projetos de pesquisa; (ii) Coordenador - usuário do ambiente que possui conhecimento específico em determinadas áreas do conhecimento, estando ligado à coordenação de algum projeto de pesquisa. Esse usuário pode contribuir na coleta, avaliação e manutenção da memória organizacional. Acessa as ferramentas de apoio ao grupo, ferramentas administrativas, ferramentas de colaboração, gerenciamento do ambiente, sistema de acesso, sistema de ajuda e projetos de pesquisa; (iii) Pesquisador - usuário do ambiente que tem acesso ao conteúdo armazenado na memória organizacional necessário à realização bem-sucedida de sua tarefa. Esse usuário pode contribuir na coleta, avaliação e manutenção da memória organizacional. Acessa as ferramentas de apoio ao grupo, ferramentas de colaboração, sistema de ajuda e projetos de pesquisa; (iv) Engenheiro do Conhecimento - usuário que gerencia questões operacionais e de monitoramento do ambiente, bem como avalia o conteúdo da memória organizacional, o uso do sistema e sua manutenção. Tem acesso a ferramentas administrativas, ferramentas de auxílio na operação do ambiente, ferramentas de colaboração, sistema de acesso, sistema de ajuda e gerenciamento do ambiente;

2. Ferramentas de colaboração - correspondem ao agrupamento das ferramentas utilizadas pela comunidade de usuários do ambiente, para interações e troca de informações. Algumas ferramentas classificam-se como acadêmicas e não acadêmicas. Entre essas ferramentas estão a ferramenta de e-mail, lista de discussão, fórum e videoconferência;

3. Ferramentas de apoio ao grupo - possibilitam o acesso a ferramentas auxiliares e de apoio aos usuários do grupo de pesquisa. Compreendem ferramentas de busca de conteúdo na memória organizacional, $F A Q$, sites, mapa de competências, instituições parceiras, guia de processos, acesso e manipulação de bibliotecas digitais e do catálogo de eventos para publicação;

4. Sistema de acesso - permite o acesso dos usuários ao ambiente por meio da autenticação por login/senha. Cada objeto de conhecimento ou serviço existente no sistema deve possuir restrições de acesso. Um usuário do sistema poderá acumular diferentes perfis que o atribuem a um determinado nível de acesso ao sistema. 0 controle de acesso deve permitir cadastrar grupos de acesso, cadastrar usuários e atribuir os serviços, bem como possibilitar ao usuário a troca de senha. Um ou mais membros do grupo de pesquisa devem possuir a responsabilidade de gerenciar o controle de acesso, a fim de que este esteja sempre atualizado;

5. Sistema de ajuda - engloba a ajuda on-line e a FAQ do ambiente, objetivando o esclarecimento de possíveis dúvidas do usuário em relação a questões operacionais do ambiente;

6. Ferramentas administrativas - permitem aos usuários o acesso a informações administrativas do ambiente por meio de mural, cronograma de reuniões, atualização sobre a situação do grupo e novidades;

7. Notificação do agente - corresponde a um conjunto de avisos emitidos pelos assistentes (agentes inteligentes) para informar o usuário a respeito do status do ambiente. Os assistentes notificam links e usuários relacionados, requisição da atualização da keyword, prazos do cronograma, novos itens de mural, itens de novidades, e-mails e outros pontos; 
8. Gerenciamento do ambiente - corresponde a um conjunto de ferramentas que permitem a administração e o gerenciamento do ambiente, tais como log de usuário, monitoramento e outros;

9. Projetos de pesquisa - possibilitam o gerenciamento e o acompanhamento dos projetos de pesquisa desenvolvidos pelos pesquisadores, em diferentes níveis de detalhamento, relativamente aos usuários envolvidos e ao conteúdo dos projetos;

10. Mapa de competências - tem a função de mapear o nível de conhecimento dos membros do grupo de pesquisa, baseando-se em um conjunto de competências essenciais pré-definidas. Esse recurso possibilita a formação de equipes motivadas, visando ao aumento da produtividade e à maximização de resultados. Pelo exposto, o ambiente suporta um amplo conjunto de ferramentas interativas, compostas de conteúdo (memória organizacional), gerência, suporte a usuários e ferramentas de colaboração e de apoio ao grupo, visando à completa gestão do conhecimento em um grupo de pesquisa.

Procura-se, então, construir um ambiente caracterizado pela existência de diferenciais competitivos marcantes, gerados principalmente por: (i) definição de uma proposta adequada para o desenvolvimento de uma infraestrutura tecnológica que forneça suporte à gestão do conhecimento em grupos de pesquisa; (ii) aplicação de tecnologias emergentes, tais como portais do conhecimento, raciocínio baseado em casos, ontologias e agentes inteligentes; (iii) definição de processos otimizados, gerando reuso.

Abaixo estão listadas, de forma mais específica, as diferentes propriedades que o ambiente deve apresentar:

1. Acesso eficiente e eficaz às informações na memória organizacional. Consiste em oferecer acesso à informação e ao conhecimento armazenados na memória organizacional, por meio de procedimentos de filtragem e recuperação de informações. Isso facilita a aquisição do conhecimento necessário à realização das atividades desenvolvidas no grupo de pesquisa;

2. Distribuição proativa do conhecimento. Refere-se à utilização de agentes inteligentes identificadores de informações e/ou conhecimentos úteis e, conforme o perfil do usuário e seu interesse, disparam essas informações para ele;

3. Recuperação eficiente e eficaz das informações. Baseada na consulta formulada por um membro do grupo, a memória organizacional deve ser pesquisada e os casos mais relevantes são retornados. Para isso, sugere-se utilizar a técnica de raciocínio baseado em casos (RBC), que consiste no uso da recuperação com base na similaridade, visando fornecer ao usuário informações relevantes;

4. Aplicações de Inteligência Artificial (IA). Várias técnicas de IA poderão ser aplicadas no ambiente, gerando eficácia no processo de suporte às ações dos usuários. Essas aplicações têm como foco principal o auxílio aos usuários desde o momento em que acessam o ambiente, buscando um determinado conhecimento, até a finalização do processo de aquisição desse conhecimento.

Para o desenvolvimento desse ambiente, foi proposta uma arquitetura modular, que pode ser visualizada na Figura 5, baseada em três níveis: nível de apresentação, nível de aplicação e nível de gerenciamento do conhecimento. 
Figura 5 - Arquitetura para o SGC em grupos de pesquisa e desenvolvimento

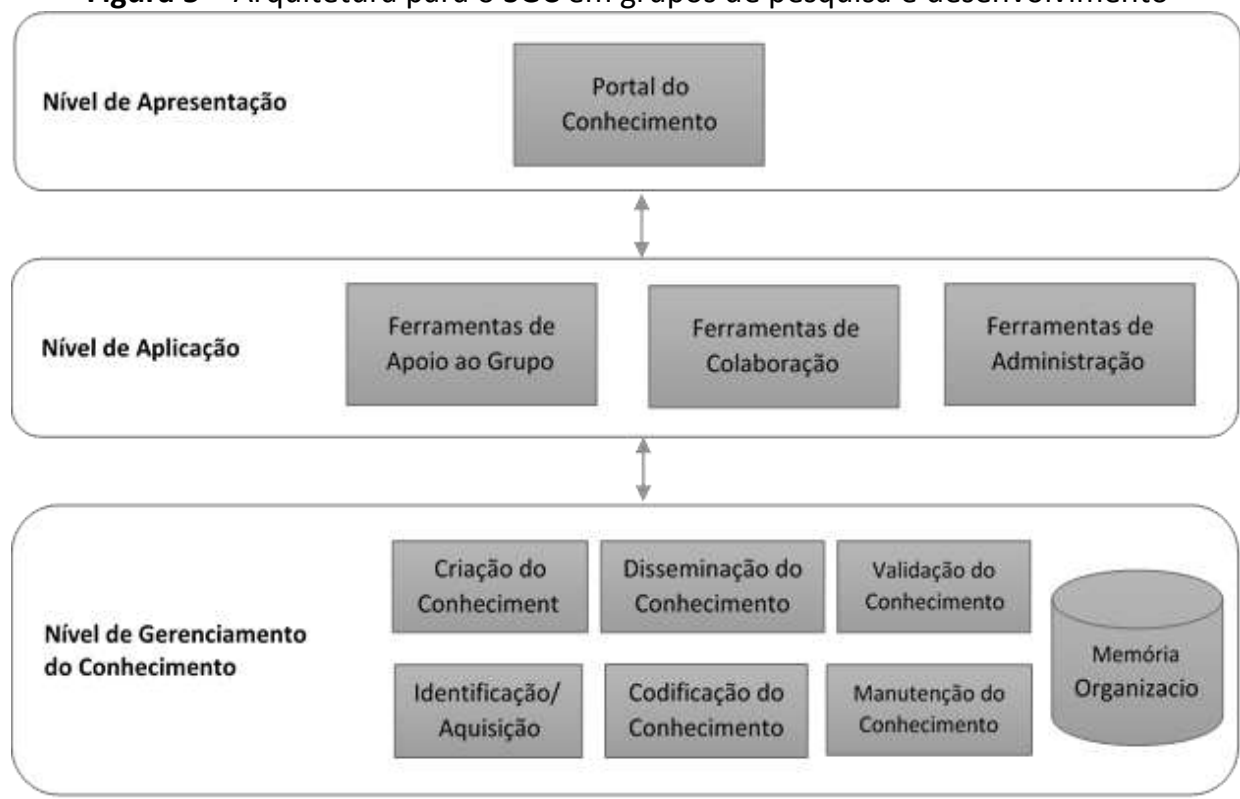

Fonte: Autoria própria

Essa arquitetura mostra-se adequada não apenas para a modularização do SGC em termos de suas funções e, consequentemente, em termos dos seus componentes tecnológicos, mas, sobretudo, para viabilizar a criação, a disseminação e o compartilhamento de conhecimentos no âmbito de um grupo de pesquisa. Para o entendimento adequado dessa arquitetura, serão explicitados cada um de seus níveis.

1. Nível de apresentação - representado pelo portal do conhecimento. Trata-se da interface que dá acesso às funcionalidades das ferramentas do nível de aplicação. Esse nível possui recursos que possibilitam utilizar o portal do conhecimento como principal meio de funcionamento, apresentar os resultados e as consultas em diversos formatos, dependendo do perfil do usuário, e acessar os serviços que estão disponíveis no nível de aplicação. O portal provê, ainda, uma interface simples e amigável para que o usuário possa acessar as informações e o conteúdo do SGC de forma personalizada. Para isso, esse portal deve atender aos seguintes objetivos: dar visibilidade da missão e dos objetivos do grupo, seus pesquisadores, suas competências e projetos de pesquisa desenvolvidos ou em desenvolvimento pelo grupo, bem como ser uma solução para pesquisa de conteúdo na memória organizacional e colaboração das equipes de projetos. A Figura 6 apresenta uma arquitetura para portais de grupos de pesquisa;

2. Nível de aplicação. Esse nível da arquitetura representa todas as aplicações que fornecem suporte a uma série de serviços para o grupo de pesquisa, tais como: ferramentas de apoio ao grupo, ferramentas de colaboração e ferramentas administrativas, entre outras;

3. Nível de gerenciamento do conhecimento. Esse nível da arquitetura é o responsável por armazenar e gerenciar o conhecimento gerado por meio da memória organizacional. A memória organizacional pode ser vista como uma estrutura de repositórios nos quais os diferentes objetos de conhecimento são armazenados, e com base neles o conhecimento pode ser recuperado. Em outras palavras, esse nível é o responsável pela gestão da memória organizacional relacionada aos grupos de pesquisa. 
Figura 6 - Visão do portal do conhecimento pelos usuários do grupo de pesquisa.

Simbolo

do Grupo

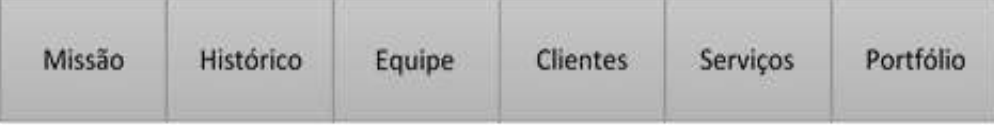

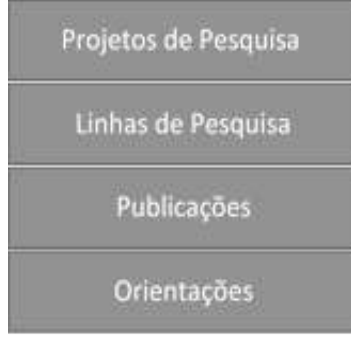

Sistema de Acesso

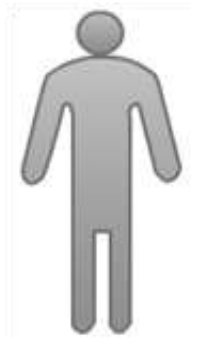

Usuário

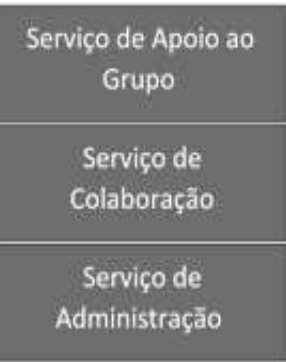

Sistema de Ajuda

Fonte: Autoria própria

Salienta-se que a abordagem adotada na formulação do modelo de SGC para grupos de $P \& D$ deve prover condições para que as implementações se adaptem às diferentes situações em que forem realizadas. A equipe de desenvolvimento deve estar ciente da necessidade de que as técnicas e tecnologias adotadas deverão suportar as propriedades distribuídas e colaborativas dos componentes da arquitetura proposta para o modelo e da independência de plataformas e arquiteturas computacionais.

\section{CONSIDERAÇÕES FINAIS}

A democratização do acesso à Educação Superior, a elevação da quantidade de matrículas, o aumento da qualidade e da proporção de mestres e doutores do corpo docente em efetivo exercício no conjunto do sistema de Educação Superior para $75 \%$ (setenta e cinco por cento) e, ainda, a elevação gradual do número de matrículas na pós-graduação stricto sensu, de modo a atingir a titulação anual de 60.000 (sessenta mil) mestres e 25.000 (vinte e cinco mil) doutores, são compromissos do governo brasileiro expressos no Plano Nacional de Educação (PNE), instituído pela Lei no 13.005/2014 (BRASIL, 2014). Os grupos de pesquisa e desenvolvimento, além de contribuírem para a própria pesquisa e para o desenvolvimento, auxiliam na formação especializada de recursos humanos nas diversas áreas do conhecimento e estão alinhados com as metas e diretrizes do PNE.

O presente artigo buscou evidenciar como um SGC, interligado em uma rede de produção do conhecimento, pode se tornar uma ferramenta eficaz de apoio gerencial no interior de uma IES. Dentro dessa perspectiva, destaca-se que a gestão do conhecimento possui um gama interessante de ferramentas para apoiar a captura, armazenamento e disseminação do conhecimento científico em organizações de P\&D, como é o caso das IES.

A fundamentação teórica revela que a melhoria do desempenho de uma IES requer o reconhecimento da informação e do conhecimento como um dos principais recursos organizacionais, o que exige modificações nas práticas administrativas correntes e na utilização da gestão do conhecimento como uma forma de possibilitar melhorias no seu desempenho.

Observa-se também a importância do aprendizado colaborativo e cooperativo em rede, como forma de incrementar potencial de inteligência coletiva dos pesquisadores pertencentes aos grupos de pesquisa e desenvolvimento. Esse fato evidencia a importância da 
definição de um modelo de sistema de gestão do conhecimento que forneça suporte às atividades do grupo, registrando as soluções adotadas para a resolução de problemas, bem como retendo e gerenciando o conhecimento de seus pesquisadores.

O SGC para grupos de P\&D tem o potencial de colocar à disposição da comunidade científica todo o capital intelectual de um determinado grupo, objetivando criar uma interação entre os vários grupos existentes em uma IES para que possam realizar parcerias e agregar valor ao desenvolvimento em ciência, tecnologia e inovação do país. Sendo assim, verifica-se a necessidade de estudar, em trabalhos futuros, as relações intergrupais, visando à formação de redes de pesquisa.

\section{REFERÊNCIAS}

AGRASSO NETO, M.; ABREU, A. F. Ferramenta de Apoio à Gestão do Conhecimento: aspectos conceituais. In: CONGRESSO BRASILEIRO DE GESTÃO DO CONHECIMENTO (KM Brasil), 3., 2002, São Paulo: UFSCar. Anais.... São Paulo, 2002a.

AGRASSO NETO, M.; ABREU, A. F. Grupos de pesquisa segundo uma abordagem sociotécnica. In: CONGRESSO BRASILEIRO DE BIBLIOTECONOMIA, DOCUMENTAÇÃO E CIÊNCIA DA INFORMAÇÃO. Fortaleza: ACB, Anais.... Fortaleza, 2002b.

BOWDITCH, J. L.; BUONO, A. F. Comportamento Grupal e Intergrupal: elementos de comportamento organizacional. São Paulo: Pioneira, 1992.

BRASIL. Plano Nacional de Educação 2014 - 2024. Centro de Documentação e Informação, Câmara dos Deputados, Brasília - 2014. Disponível em: http://www.observatoriodopne.org.br/uploads/reference/file/439/documento-referencia.pdf. Acesso em: 22 jun. 2016.

BRAZIER, F.M.T.; WIJNGAARDS, N.J.E. An instrument for a purpose driven comparison of modelling frameworks. Knowledge Acquisition, Modeling and Management, v. 1319, p. 323328, Lecture Notes in Computer Science (Série). Springer, 2005.

BREUKER, J.; VAN DE VELDE, W. CommonKADS Library for Expertise Modelling: Reusable Problem Solving Components. Amsterdan: IOS Press, 1994.

CINALLI, D.; MARTÍ, L.; SANCHEZ-PI, N.; GARCIA, A.C.B. Integrating Collective Intelligence into Evolutionary Multi-Objective Algorithms: Interactive Preferences. In: LATIN AMERICA CONGRESS ON COMPUTATIONAL INTELLIGENCE (LA-CCI). Curitiba: IEEE, 2015. Proceedings... Curitiba, 2015.

CONSELHO NACIONAL DE DESENVOLVIMENTO CIENTífICO E TECNOLÓGICO. Diretório dos Grupos de Pesquisa. Brasília, 2016. Disponível em: http://lattes.cnpq.br/web/dgp. Acesso em: 08 mai. 2016.

FENSEL, D.; MOTTA, E. Structured Development of Problem Structuring Methods. IEEE Transactions on Knowledge and Data Engineering, v. 13, n. 16, p. 913-932, 2001.

FERNANDES, A. M. R. Inteligência Artificial: noções gerais. 1. ed., Visual Books, 2003. 
HEVNER, A. R.; MARCH, S. T.; PARK, J.; RAM, S. Design science in information systems research. MIS Quarterly, vol. 28, n. 1, p. 75-105, 2004.

GARCIA, C. L. S.; VALENTIM, M. L. P. Gestão do Conhecimento Científico: proposta de um modelo para a área de Ciência da Informação da Universidade Estadual Paulista (Unesp). Tendências da Pesquisa Brasileira em Ciência da Informação, v. 7, n. 1, 2014.

GONÇALVES, A.L. Utilização de Técnicas de Mineração de Dados na Análise dos Grupos de Pesquisa no Brasil. Dissertação (Mestrado em Engenharia de Produção - Programa de PósGraduação em Engenharia de Produção), UFSC, agosto, Florianópolis, 2000.

INTERNATIONAL STANDARD ORGANIZATION. ISO 10006: Quality management - Guidelines to quality in Project management. 2003.

LÉVY, P. Collective Intelligence. New York: Plenum, Harper Collins, 1997.

LÉVY, P. As Inteligências Coletivas. Palestra. Sesc-SP. 2002. Disponível em: http://www2.sescsp.org.br/sesc/hotsites/pierre levy/. Acesso em: 10 abr. 2016.

LÉVY, P. From social computing to reflexive collective intelligence: The IEML research program. Information Sciences, vol. 180, n. 1, p. 71-94, 2010.

LICHTNOW, D. Desenvolvimento e implementação de um protótipo de ferramenta para gestão do conhecimento em grupos de pesquisa. Dissertação (Mestrado em Ciência da Computação) - Programa de Pós-graduação em Ciência da Computação, Departamento de Informática, UFSC, Florianópolis, 2001.

MEZA, J.; MONGUET, J.M.; GRÍMON, F.; TREJO, A. Fostering Collective Intelligence Education. In: Vicenti, G.; Bucciero, A.; Carvalho, C.V. (eds.) Lecture Notes of the Institute for Computer Sciences, Social Informatics and Telecommunications Engineering, v. 160, p. 165-172, 2015.

PEFFERS, K. E. N.; TUUNANEN, T.; ROTHENBERGER, M. A.; CHATTERJEe, S. A Design Science Research Methodology for Information Systems Research. Journal of Management Information Systems, v. 24, n.3, p. 45-77, 2007.

ROMÃO, W. Descoberta de Conhecimento Interessante em Banco de Dados sobre Ciência e Tecnologia. Tese (Doutorado em Engenharia de Produção) - Programa de Pós-Graduação em Engenharia de Produção, UFSC, Fevereiro, Florianópolis, 2002.

SECUNDO, G.; DUMAY, J.; SCHIUMA, G; PASSIANTE, G. Managing intellectual capital through a collective intelligence approach. Journal of Intellectual Capital, v. 17 n. 2, p. $298-319,2016$.

SILVA, E. V.; MOSCONI, E. P.; SILVA, S. Experiências em gestão do conhecimento em um grupo de pesquisa multidisciplinar sobre desenvolvimento de produtos. In: INTERNATIONAL SYMPOSIUM ON KNOWLEDGE MANAGEMENT, $6^{\text {th }}$., Curitiba: PUC-PR, 2003. Anais.... Curitiba, 2003.

STUDER, R.; BENJAMINS, V, R.; FENSEL, D. Knowledge Engineering: Principles and Methods. Data \& Knowledge Engineering, v. 25, n. 1-2, p. 161-197, 1998. 
TJORNBO, O. Can Collective Intelligence Produce Social Innovation? In: Nicholls, A.; Simon, J.; Gabriel, M (Orgs.). New Frontiers in Social Innovatios Research. Palgrave Macmillan, p. 192211, 2016. 